Discrete Comput Geom 29:139-152 (2003)

DOI: $10.1007 / \mathrm{s} 00454-002-0730-2$

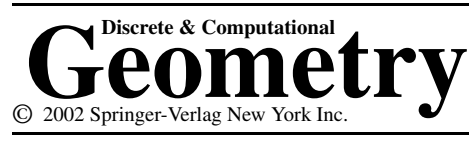

\title{
On the Identification of Sets of Points in the Square Lattice*
}

Iiro Honkala and Tero Laihonen

Department of Mathematics, University of Turku, 20014 Turku, Finland

\{honkala,terolai\}@utu.fi

\begin{abstract}
Identifying codes in the square lattice are considered. The motivation for these codes is the following: if a multiprocessor system is modelled by the square lattice, then we can locate faulty processors in the system with the aid of identifying codes. Constructions, some of which are optimal, are given.
\end{abstract}

\section{Introduction}

We consider the situation in which each vertex of an undirected graph $G=(V, E)$ contains a processor, and $E$ gives the set of links between the processors. In this paper we take as our graph the two-dimensional square lattice $T$ with the vertex set $V=\mathbb{Z}^{2}$ and edge set $E=\{\{u, v\}: u-v=( \pm 1,0)$ or $u-v=(0, \pm 1)\}$.

We wish to perform a fault diagnosis on the system. We choose a suitable subset $C \subseteq V$ and let each processor in $C$ check all the processors within distance $r$ in the graph (where $r$ is a given integer). Here the distance $d(u, v)$ between the vertices $u$ and $v$ means the number of edges on any shortest path from $u$ to $v$. Each of these processors then sends one bit of information to us, according to whether it has detected anything out of order or not. Based on these answers we wish to obtain some information on $F$, the set of all the vertices in which the processors are malfunctioning.

The study of such codes was originated by Karpovsky et al. [9].

Denote by $Q_{n}$ the set of vertices $(x, y) \in V$ with $|x| \leq n$ and $|y| \leq n$. Then the density of $C$ is defined as

$$
D(C)=\limsup _{n \rightarrow \infty} \frac{\left|C \cap Q_{n}\right|}{\left|Q_{n}\right|} .
$$

In this paper we only consider the case $r=1$.

* The research of the authors was supported by the Academy of Finland under Grants 44002 and 46186. 
Section 2 deals with the variant in which we have the additional initial assumption that $|F| \leq k$, and the answers obtained from the vertices in $C$ completely identify $F$, i.e., its cardinality and all its elements. It turns out that this approach only works for $k \leq 3$. It is known that for $k=1$, the optimal density is at least $\frac{15}{43} \approx 0.3488$ (see [4] and [5]) and at most $\frac{7}{20}=0.35$ (see [3]). We show that for $k=2$, the optimal density is $\frac{1}{2}$ and for $k=3$ it is 1 . For the values $k=1$ and $r \leq 7$ consult [2]; in general, it is known (see [1]) that for $k=1$ and all $r$, the density must be at least $3 /(8 r+4)$, and there is a construction with density $2 /(5 r)$ if $r$ is even, and $2 r /\left(5 r^{2}-2 r+1\right)$ if $r$ is odd (see [8] and [1]).

In Section 3 we consider the case in which $F$ is assumed to be finite, but there is no a priori upper bound on its size. In this case we would like to identify at least one element of $F$. We prove that this is only possible if the density of $C$ equals 1 .

In Section 4 we consider the case in which we have the initial assumption that $|F| \leq k$, but it is only required that based on the answers from the elements in $C$, we can find at least one element of $F$. For any $k$, we give a construction with density $k /(k+1)$. For $k=2$, we give a construction with density $\frac{4}{9}$, and for $k=3$, a construction with density $\frac{7}{12}$.

In the case when the graph $G$ is the Hamming space, the problems of Sections 2 and 4 have been considered, for instance, in [9], [7], [12] and [10].

The set $C$ is called $a$ code and its elements codewords. Denote $B_{r}(v)=\{x \in$ $V: d(x, v) \leq r\}$. If $u, v \in V$, and $d(u, v) \leq 1$, we say that $u$ covers $v$ (and vice versa). A code $C$ is called $r$-dominating if for every $v \in V$, there is a vertex $u \in C$ such that $d(u, v) \leq r$.

For a closely related problem of locating-dominating sets, we refer to [14], [13], [11], and [6]. A code $C \subseteq V$ is called a locating-dominating set if the sets $B_{1}(v) \cap C$ are nonempty and different for $v \in V \backslash C$, i.e., only for the non-codewords $v$. It has been shown by Slater [14] that $\frac{3}{10}$ is the smallest possible density of a locating-dominating set in the square lattice.

\section{On Identifying Codes}

In this section we explore the density of $(1, \leq k)$-identifying codes when $k \geq 2$.

Let $C \subseteq V$. Denote for any $X \subseteq V$,

$$
I_{r}(X)=\left(\bigcup_{v \in X} B_{r}(v)\right) \cap C .
$$

We further denote $I_{1}(X)=I(X)$ and $I_{r}\left(\left\{x_{1}, \ldots, x_{s}\right\}\right)=I_{r}\left(x_{1}, \ldots, x_{s}\right)$.

Definition 1. Let $r$ and $k$ be nonnegative integers. A code $C \subseteq V$ is called $(r, \leq k)$ identifying if $I_{r}\left(X_{1}\right) \neq I_{r}\left(X_{2}\right)$ for all $X_{1}, X_{2} \subseteq V$ where $X_{1} \neq X_{2}$ and $\left|X_{1}\right|,\left|X_{2}\right| \leq k$.

Theorem 1. The smallest density of a $(1, \leq 2)$-identifying code in the square lattice is $\frac{1}{2}$. 


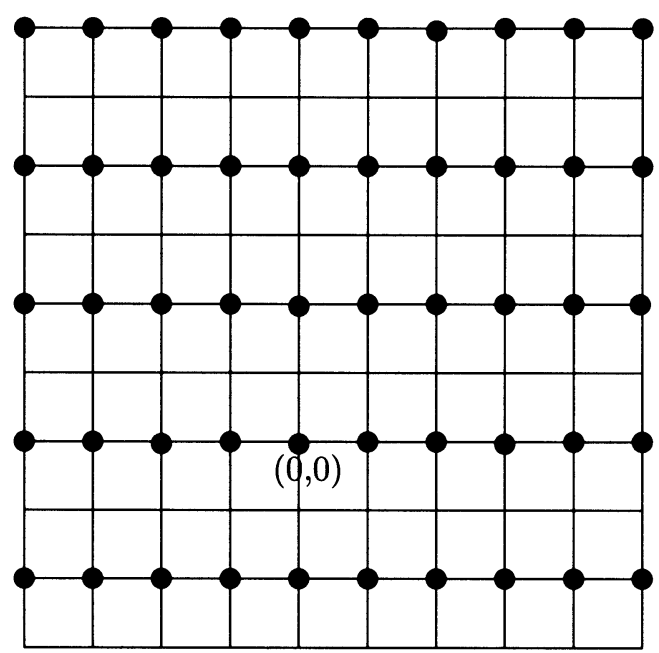

Fig. 1. A $(1, \leq 2)$-identifying code (part). Circles are codewords.

Proof. (Lower bound) Let $C \subseteq V$ be a $(1, \leq 2)$-identifying code. Obviously every point in $V$ is covered by at least one codeword of $C$. If $x \notin C$, then $|I(x)| \geq 2$, since if $I(x)=\{c\}$, then $I(c)=I(x, c)$, a contradiction. On the other hand, if $x \in C$, then $|I(x)| \geq 3$. Indeed, if $I(x)=\{x\}$, then $I(a)=I(x, a)$ for any $a \in B_{1}(x) \backslash\{x\}$, and the case $I(x)=\{x, c\}(x \neq c)$ implies that $I(c)=I(x, c)$, a contradiction again.

We count in two ways the number $N$ of pairs $(c, x)$ where $c \in C, x \in Q_{n}$ and $d(x, c) \leq 1$. By the discussion above

$$
5\left|Q_{n} \cap C\right| \geq N \geq\left|Q_{n-1} \cap C\right|+2\left|Q_{n-1}\right| .
$$

Since $\left|Q_{n-1}\right|=\left|Q_{n}\right|-8 n$ and $\left|Q_{n-1} \cap C\right| \geq\left|Q_{n} \cap C\right|-8 n$, we get

$$
\frac{\left|Q_{n} \cap C\right|}{\left|Q_{n}\right|} \geq \frac{1}{2}-\frac{6 n}{\left|Q_{n}\right|}
$$

and hence the density of $C$ is at least $\frac{1}{2}$.

(Upper bound) The code $C=\{(i, j) \in V: j \equiv 0 \bmod 2\}$ illustrated in Fig. 1 is $(1, \leq 2)$-identifying. This will be shown next.

Let $x$ be a point in $V$. If $x=(i, j) \in C$, then $(i-1, j)$ and $(i+1, j)$ belong to $I(x)$. Similarly, if $x=(i, j) \notin C$, then $(i, j-1),(i, j+1) \in I(x)$. In both cases $I(x)$ cannot be covered by a single point other than $x$. Firstly, this shows that $C$ is $(1, \leq 1)$ identifying. Moreover, this yields that $I(F) \neq I(\Lambda)$ for any pair $F=\{x, y\} \subseteq V$ and a singleton set $\Lambda=\{\alpha\} \subseteq V$. This follows since we may assume that $x \neq \alpha$ and thus $\alpha$ cannot cover $I(x)$.

Assume next that $F=\{x, y\} \subseteq V$ and $\Lambda=\{\alpha, \beta\} \subseteq V$ where $x \neq y$ and $\alpha \neq \beta$. Without loss of generality we can assume that $x \notin \Lambda$ and $\alpha \notin F$. It remains to show that $I(F) \neq I(\Lambda)$ for any such $F$ and $\Lambda$.

Let first $x \notin C$ and without loss of generality let $x=(0,1)$. By the discussion above it is enough to examine the cases where $(0,2)$ is covered by $\alpha$ and $(0,0)$ by $\beta$. 
Notice that $\beta$ cannot cover any point whose ordinate is more than one. There are three possibilities we have to consider, namely, $\alpha$ is one of the points $a=(-1,2), b=(0,2)$ or $c=(0,3)$ (the case $\alpha=(1,2)$ is symmetric to $(-1,2)$ ). Let first $\alpha=a$. This implies that $(-2,2) \in I(\Lambda)$ and $a \in I(\Lambda)$ and thus $y$ must cover them both but not $(-3,2)$ and hence $y=\alpha$ which is impossible. Suppose then $\alpha=b$. Now $a \in I(\Lambda)$ and $(1,2) \in I(\Lambda)$ and hence again $y=\alpha$. Assume next that $\alpha=c$. Then $(0,4) \in I(\Lambda)$ and $y$ must cover it. However, then $y \neq \alpha$ covers also another codeword of $C$ which is not contained in $I(\Lambda)$.

Suppose then that $x \in C$ and $x=(0,0)$. It suffices to check the cases where $\alpha$ covers $(-1,0)$ and $\beta$ covers $(1,0)$. It is enough to study the situations where $\alpha$ equals $a=(-2,0), b=(-1,0)$ or $c=(-1,1)$. Let first $\alpha=a$. From the facts that $(-3,0) \in$ $I(\Lambda)$ and $\alpha \in I(\Lambda)$ and that the point $y$ must cover them but not $(-4,0)$ we can deduce $\alpha=y$ which is not allowed. Suppose then that $\alpha=b$. This implies that $a \in I(\Lambda)$ and hence $y$ has to cover it but nothing else outside $I(\alpha)$ that $\beta$ does not cover. Thus $\alpha=y$ again. Let then $\alpha=c$. Now $y$ must cover $(-1,2)$ without covering anything outside $I(\alpha)$ which is not covered by $\beta$. Consequently, $y=\alpha$.

This leads to the conclusion that $C$ is $(1, \leq 2)$-identifying. Clearly, the density of $C$ is $\frac{1}{2}$.

We now consider the case $k=3$. Notice that there cannot exist $(1, \leq k)$-identifying codes if $k \geq 4$ due to the fact that even if $C=V$, we have the contradiction $I((-1,-1)$, $(0,1),(1,1))=I((-1,-1),(0,0),(0,1),(1,1))$. It is easy to check that if $C$ is $(1, \leq$ $3)$-identifying, then $|I(x)| \geq 5$. Therefore, we know that if there exists a $(1, \leq 3)$ identifying code, then the only choice is $C=V$.

In fact, the code $C=V$ is $(1, \leq 3)$-identifying. Evidently, it is $(1, \leq 2)$-identifying because $C=V$ is a supercode of the code of Fig. 1. In the square lattice the intersection of two spheres of radius one contains at most two elements provided that the centers are distinct. Since every point $x \in V$ is now covered by five codewords, we need at least three points other than $x$ to cover $I(x)$. Any point or a pair cannot cover the same set of codewords as a triplet; namely, in a triplet there exists a point which is not included in the pair or the singleton set. Consequently, we only need to check that $I(F) \neq I(\Lambda)$ for any distinct triplets $F=\{x, y, z\}$ and $\Lambda=\{\alpha, \beta, \gamma\}$.

Naturally, we can assume that $x \notin \Lambda$ and $\alpha \notin F$. Suppose without loss of generality that $x=(0,0)$. By the discussion above we may assume that $\alpha$ is one of the following points $a=(0,2), b=(0,1)$ or $c=(1,1)$. Let $\alpha=a$ (the two other cases go analogously). Then $(0,3) \in I(\Lambda)$ and therefore $y$ or $z$ must cover it, say $y$ does. However, hence $I(y)$ contains a point whose ordinate is at least four, but no such point can be in $I(\Lambda)$ since $\beta$ and $\gamma$ must cover $I(x) \backslash\{(0,1)\}$. Thus we reach the following conclusion.

Theorem 2. The smallest density of a $(1, \leq 3)$-identifying code in the square lattice equals one.

Of course, if one must take the whole lattice, then the distance $r=0$ is a better choice than $r=1$ in the sense that in that case the set of faulty processors of any cardinality can be immediately found. 


\section{Universally Identifying Codes}

The purpose of this section is to consider codes with the following identification property.

Definition 2. Let $G=(V, E)$ be an infinite graph. A 1-dominating subset $C \subseteq V$ is called a universally (1-)identifying code if it has the following property: if $S \subseteq V$, and there is a finite subset $F \subseteq V, F \neq \emptyset$, such that $S=I(F)$, then there is a vertex $v_{S} \in V$ which is contained in every finite subset $F \subseteq V$ such that $S=I(F)$.

The meaning of the definition is clear: a universally identifying code has the property that-assuming that the number of malfunctioning processors is finite-it conveys enough information so that we can locate at least one of the malfunctioning processors, fix it and continue the process.

Example 1. In the infinite square lattice $T=(V, E)$, the whole vertex set $V$ is universally identifying. Indeed, given any set $S \subseteq V$ such that $S=I(F)$ for some (unknown) $F \neq \emptyset$, we can always determine at least one element of $F$ in the following way. Define a function $f: \mathbb{Z}^{2} \rightarrow \mathbb{Z}$ by $f(i, j)=2 i+j$. Since $S$ is itself finite, we can choose a vertex $v=(i, j) \in S$ with the highest value of $f$. Then $(i, j+1) \notin S$ and $(i+1, j-1) \notin S$. Consequently, $(i, j) \notin F,(i, j+1) \notin F,(i+1, j) \notin F,(i, j-1) \notin F$, and therefore $(i, j) \in S$ implies that $(i-1, j) \in F$.

Example 2. In the infinite square lattice $T=(V, E)$, the set $C=V \backslash\{(0, j): j \in \mathbb{Z}\}$ is universally identifying. Clearly, $I(v) \neq \emptyset$ for all $v \in V$. Assume that $S=I(F)$ for some nonempty $F$. The set $S$ is nonempty, and by symmetry we can assume that it contains at least one vertex $v=(i, j)$ with $i>0$ (if not, reflect everything with respect to the line $x=0)$. If we take $v=(i, j)$ to be the vertex in $S$ with the largest value of $f$ in the half-plane $x>0$, we again see that $(i-1, j) \in F$.

Unfortunately, we cannot do much better than in the two previous examples.

Theorem 3. Assume that $C$ is a universally identifying code in the infinite square lattice. Then the density of $C$ is 1 .

Proof. Consider the set $N$ of non-codewords, which, by assumption, has a positive density.

Assume that $N$ contains the four points

$$
(a, b),(a+s, b-s),(a+t, b+t),(a+s+t, b-s+t)
$$

for some $a, b$ and $s \geq 2, t \geq 2$ (see Fig. 2). These four points form a rectangle with all sides having slopes 1 or -1 . We denote by $E$ the set of all lattice points in the interior (marked as squares in Fig. 2), and by $B$ the set of all lattice points in the boundary (marked as circles). We see that whenever $F$ consists of all the points in $E$ except one, then $I(F)=(B \cup E) \cap C$ : here we need the fact that the corner points do not belong to $C$ and that $s \geq 2$ and $t \geq 2$. 


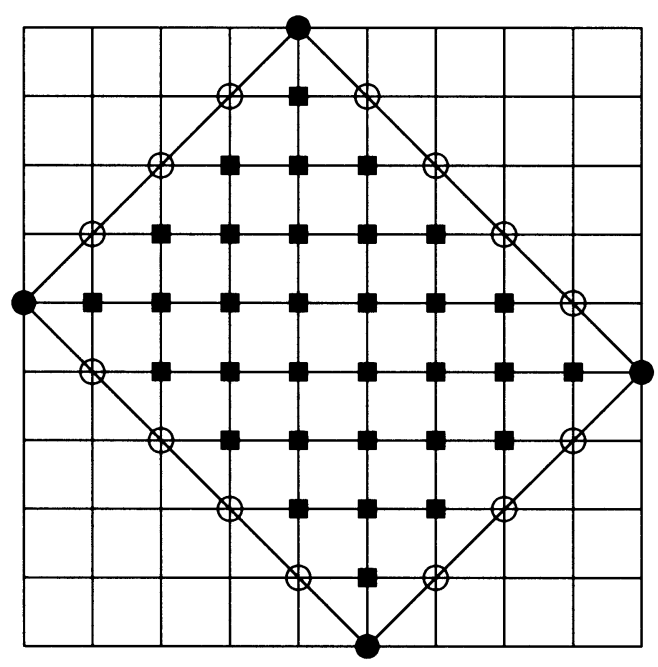

Fig. 2. A forbidden pattern.

It suffices to show that such a forbidden pattern (1) always exists in $N$. For that purpose, we partition the original square lattice $T$ into eight subsets

$$
T(v)=v+\{i(2,2)+j(2,-2): i, j \in \mathbb{Z}\}
$$

for $v \in L:=\{(0,0),(1,0),(2,0),(3,0),(1,1),(2,1),(1,-1),(2,-1)\}$. The sets $T(0,0)$ and $T(1,1)$ are shown in Fig. 3. If we denote

$$
Q_{n}(v)=v+\{i(2,2)+j(2,-2):|i| \leq n,|j| \leq n\}
$$

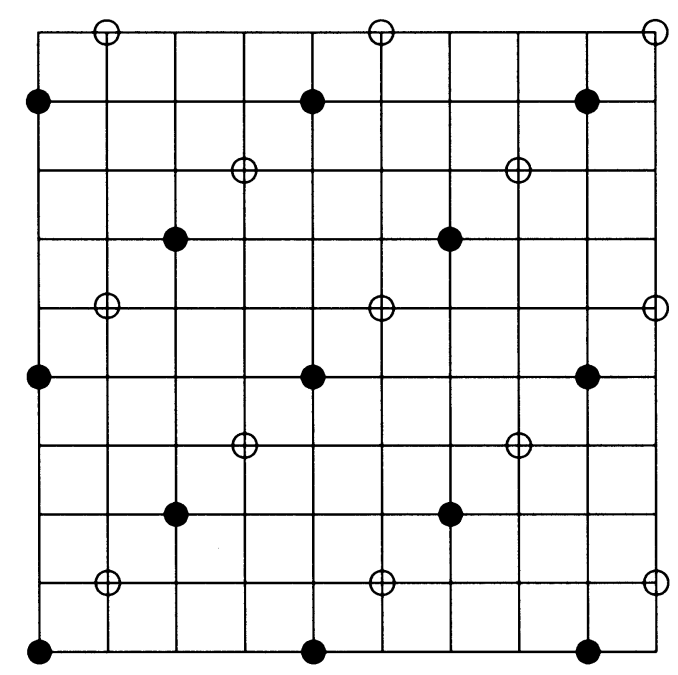

Fig. 3. The subsets $T(0,0)$ and $T(1,1)$. 
and the density of $N$ in $T(v)$ by

$$
D(v)=\limsup _{n \rightarrow \infty} \frac{\left|N \cap Q_{n}(v)\right|}{\left|Q_{n}(v)\right|},
$$

then since the density of $N$ in $T$ is positive, at least one of the densities $D(v)$ is also positive. This is clear, because

$$
Q_{n} \subseteq Q_{2 n} \subseteq \bigcup_{v \in L} Q_{n}(v),
$$

where $Q_{n}=\{(i, j):|i| \leq n,|j| \leq n\}$.

Without loss of generality, $D(0,0)$ is positive, and we now get the result from the following lemma.

Lemma 1. In the infinite square lattice a subset with positive density contains four points which are the corners of a rectangle whose sides are parallel to the coordinates axes.

Proof. Assume that $X$ is a subset of the square lattice which does not have any four such points. We show that the density of $X$ is 0 .

Consider the set $Q_{n}=\{(i, j):|i| \leq n,|j| \leq n\}$. We can view $X \cap Q_{n}$ as a bipartite graph $G$ with the bipartition $A=\left\{a_{-n}, \ldots, a_{n}\right\}, B=\left\{b_{-n}, \ldots, b_{n}\right\}$ and the edge set $E(G)=\left\{\left\{a_{i}, b_{j}\right\}:(i, j) \in X\right\}$.

If $N\left(a_{i}\right)$ denotes the set of neighbours of $a_{i}$, for $i=-n, \ldots, n$ and $d_{i}=\left|N\left(a_{i}\right)\right|$, the requirement that $X$ does not contain any such forbidden pattern implies that no two vertices $a_{i}$ and $a_{j}$ can have two neighbours in common. Hence by the Cauchy-Schwartz inequality

$$
\left(\begin{array}{c}
2 n+1 \\
2
\end{array}\right) \geq \sum\left(\begin{array}{c}
d_{i} \\
2
\end{array}\right) \geq \frac{1}{2(2 n+1)}\left(\sum d_{i}\right)^{2}-\frac{1}{2}\left(\sum d_{i}\right) .
$$

Because $\sum d_{i}=\left|X \cap Q_{n}\right|$, we see that $\left|X \cap Q_{n}\right|=O\left(n^{3 / 2}\right)$, and therefore $X$ has density 0 . In fact, we are just using the well-known fact that if a graph with $K$ vertices has no 4-cycles, then it has at most $O\left(K^{3 / 2}\right)$ edges.

\section{Weakly Identifying Codes}

Even though there do not exist $(1, \leq k)$-identifying codes when $k \geq 4$, we can still utilize weakly identifying codes to find malfunctioning processors from the square lattice. If $k=2$ or $k=3$, then using weakly $(1, \leq k)$-identifying codes instead of $(1, \leq k)$ identifying codes we can locate the faulty processors with a code that has a smaller density. The price we have to pay is that we must be prepared to repeat the process of getting replies from the codewords (at most) $k$ times. The concept of weak identification was introduced in [7]. 


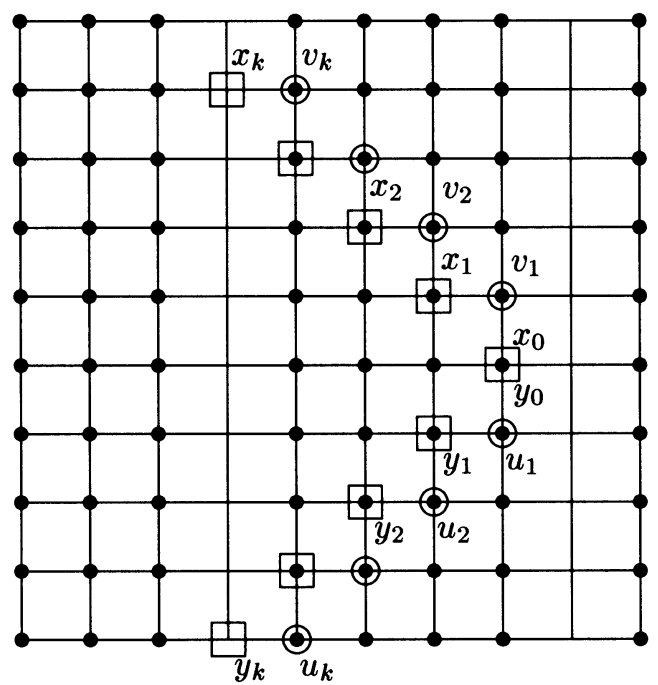

Fig. 4. A construction of a weakly $(1, \leq k)$-identifying code for $k=4$.

Definition 3. Let $G=(V, E)$ be an infinite graph. An $r$-dominating subset $C \subseteq V$ is called a weakly $(r, \leq k)$-identifying code if it has the following property: if $S \subseteq V$, and there is a finite subset $F \subseteq V$ of cardinality at most $k, F \neq \emptyset$, such that $S=I_{r}(F)$, then there is a vertex $v_{S} \in V$ which is contained in every subset $F \subseteq V(|F| \leq k)$ such that $S=I_{r}(F)$.

The following theorem shows that for every $k \geq 2$ there exists a weakly $(1, \leq k)$ identifying code with density smaller than 1 .

Theorem 4. There is a weakly $(1, \leq k)$-identifying code in the infinite square lattice with density $k /(k+1)$.

Proof. The construction consists of simply leaving out every $(k+1)$ st column of vertices, see Fig. 4.

Let $F$ be an arbitrary set consisting of at most $k$ vertices, and assume that we know $I(F)$ (but not $F$ ).

Assume that $v_{1}=(a, b)$ is the point of $I(F)$ with the highest value of the ordinate among the points with the highest value of the abscissa. From Example 1 we know that if for some $i$ and $j,(i, j) \in I(F),(i, j+1) \notin I(F)$ and $(i+1, j-1) \notin I(F)$, then $(i-1, j) \in F$. This implies that we can assume that none of the vertices in the $(b+1)$ st column are in our code. Clearly, $x_{1}=(a-1, b) \in F$ or $x_{0}=(a, b-1) \in F$. If $v_{2}=(a-1, b+1) \notin I(F)$, then we are done (because then $x_{1} \notin F$ and hence $\left.x_{0} \in F\right)$; hence we can only consider the patterns with $v_{2} \in I(F)$. We can assume that of the points in $I(F), v_{2}$ has the highest value of the ordinate in its column: otherwise, we can apply the argument of Example 1 to the point of $I(F)$ with the highest value of the ordinate in this column. By repeating the previous argument, $v_{2} \in I(F)$ now implies 
that $x_{2}=(a-2, b+1) \in F$ or $x_{1} \in F$. Continuing in the same way we see that for each $i=1,2, \ldots, k$, it suffices to consider the patterns in which $v_{i}=v_{1}+(-i+1, i-1)$ is the point of $I(F)$ with the highest value of the ordinate in its column. Again, $v_{i} \in I(F)$ then implies that $x_{i}=v_{i}+(-1,0) \in F$ or $x_{i-1}=v_{i}+(0,-1) \in F$.

If $(a, b-2) \notin I(F)$, then $x_{0} \notin F$ and consequently we know that $x_{1} \in F$. So assume that $(a, b-2) \in I(F)$.

Denote by $u_{1}$ the point of $I(F)$ with the smallest value of the ordinate in the $b$ th column (so $u_{1}=(a, b-2)$ or below it), and $y_{0}=u_{1}+(0,1)$.

By symmetry, going down instead of going up, we see that it suffices to consider the patterns in which for each $i=1,2, \ldots, k, u_{i}=u_{1}+(-i+1,-i+1)$ is the point of $I(F)$ with the smallest value of the ordinate in its column, and then $u_{i} \in I(F)$ implies that $y_{i}=u_{i}+(-1,0) \in F$ or $y_{i-1}=u_{i}+(0,1) \in F$.

However, $|F| \leq k$ implies that if $k$ is even, then $x_{2 i-1} \in F$ and $y_{2 i-1} \in F$ for all $i=1,2, \ldots, k / 2$, and if $k$ is odd, then $x_{0}=y_{0} \in F$ and $x_{2 i} \in F$ and $y_{2 i} \in F$ for all $i=1, \ldots,(k-1) / 2$.

Theorem 5. The code

$$
C=\{(i, j): i \equiv 1 \bmod 2 \text { and } j \not \equiv i+4 \bmod 9\}
$$

is weakly $(1, \leq 2)$-identifying and has density $\frac{4}{9}$.

Proof. It is easy to verify that the code $C$ illustrated in Fig. 5 is $(1, \leq 1)$-identifying. We show that based on $I(F)$ we can always find (at least) one of the faulty processors in $F$ if $|F| \leq 2$. Obviously $I(F)$ is empty if and only if $F$ is empty. We classify the patterns $I(F)$ in terms of their leaders: we define the leader of $I(F)$ to be the unique point in $I(F)$ with the highest value of the ordinate among the points with the highest value of the abscissa. We denote the leader by $c$ throughout the proof.

The codeword $c$ is one of the eight codewords in a tile (inside the dashed line) given in Fig. 5. Without loss of generality we may assume that $c$ is one of the codewords in $\{(9,5),(9,6), \ldots,(9,12)\}$. Given $c=(i, j)$ we denote $a=(i-1, j)$ and $b=(i, j-1)$.

(i) Let first the leader $c=(9,8)$ (the cases $c \in\{(9,9),(9,10)\}$ go in exactly the same way). By the definition of $c$, there are two positions where one of the faulty processors must be, namely, at $a$ or $b$. If $(7,8) \notin I(F)$, then $b \in F$. Assume that $(7,8) \in I(F)$. If now $(9,6)$ or $(9,7)$ does not belong to $I(F)$, then $a \in F$. Suppose then that these points are in $I(F)$. One element of $F$ covers $(7,8)$. If $(9,5) \notin I(F)$, we know that $b \in F$, and if $(9,5) \in I(F)$, we obtain $a \in F$ (and $(9,6) \in F)$.

(ii) Let then $c=(9,7)$. The beginning of this case goes as (i) up to the point where we can assume that the points $(9,5),(9,6)$ and $(7,7)$ belong to $I(F)$. If now none of the points $(7,8),(5,7)$ or $(7,6)$ lies in $I(F)$, then $a \in F$. If $I(F)$ contains at least one of these points, then $b \in F$ due to the fact that these point(s) in $I(F)$ must be covered by an element in $F$ but the codewords $(9,5)$ and $c$ still have to be covered by one point of $F$.

(iii) Suppose $c=(9,5)$. We may assume that $(7,5)$ and $(9,3)$ are contained in $I(F)$. If none of the points $(7,6),(5,5)$ and $(7,4)$ belongs to $I(F)$, we get $a \in F$. Assume that at least one of them does. Now the points $c$ and $(9,3)$ must be covered by a single point in $F$ and hence $b \in F$. 


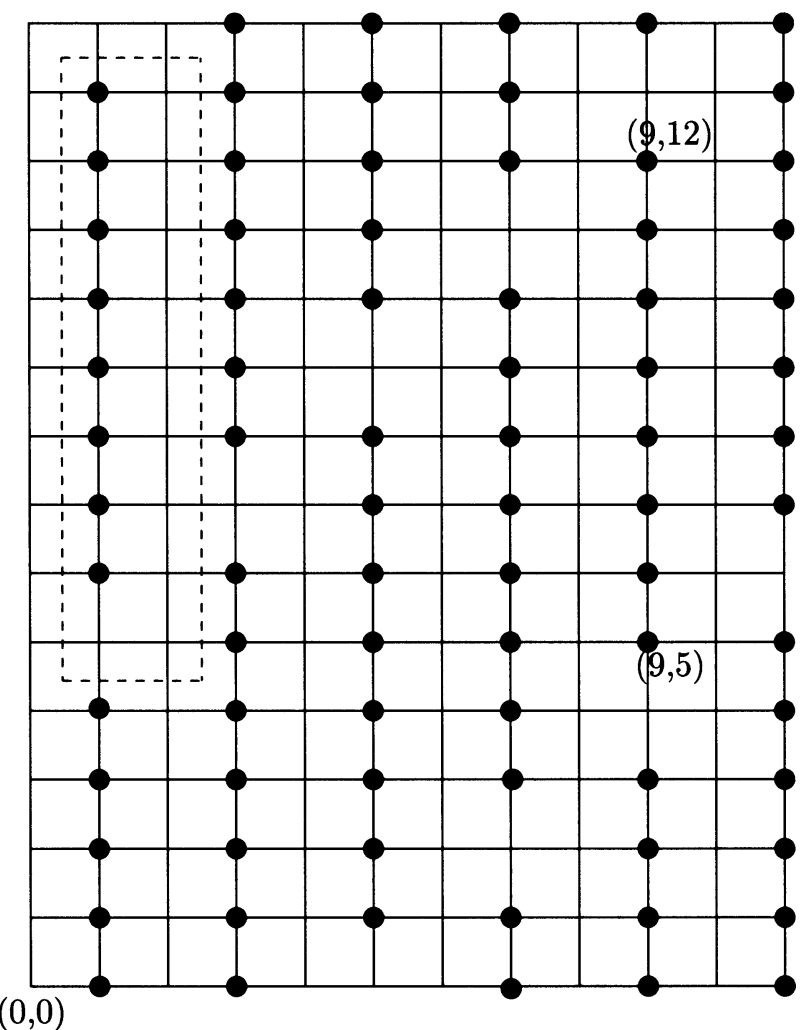

Fig. 5. A weakly $(1, \leq 2)$-identifying code (part). Codewords are denoted by circles.

(iv) Assume that $c=(9,11)$. If $b \notin I(F)$ or $y=(9,9) \notin I(F)$, then $a \in I(F)$. Suppose that both of them are contained in $I(F)$. If $f=(9,8) \notin I(F)$, we get that $b \in F$. Let then $f \in I(F)$. If neither $(9,7)$ nor $(7,8)$ belongs to $I(F)$, it is clear that $y \in F$. Assume that one of these two is in $I(F)$. Hence there is only one point in $F$ that should cover both $c$ and $b$ and this leads to the conclusion that $b \in F$.

In the last two cases we denote by $c^{\prime}$ the codeword, which is among the points with the highest value of the abscissa in $I(F)$, and has the smallest value of the ordinate such that starting from it the consecutive points up to $c$ are either in $I(F)$ or in $V \backslash C$.

(v) Let the leader $c=(9,12)$. Then $a, b$ or $c$ belongs to $F$. Suppose first that $c=c^{\prime}$. This yields $a \in F$. Suppose that $c^{\prime}=b$. Let $f=(7,12)$. If $f \notin I(F)$, one finds out that $c \in F$. Assume therefore that $f \in I(F)$. If none of the points $(7,13),(5,12)$ or $(7,10)$ is contained in $I(F)$, we immediately deduce that $a \in F$. Let then one of these be in $I(F)$. Then $c \in F$ due to the fact that only one point of $F$ is supposed to cover both $c$ and $b$.

Assume next that $c^{\prime}=(9,10)$. Denote $f=(7,10)$. If $f \notin I(F)$, we obtain $b \in F$. Suppose that $f \in I(F)$. If none of the points $x_{1}=(7,12), x_{2}=(5,10)$ and $x_{3}=(7,9)$ belongs to $I(F)$, then evidently $(8,10) \in F$. Suppose first that $x_{2}$ or $x_{3}$ is in $I(F)$. Now all the words $c, b$ and $c^{\prime}$ must be covered by one element in $F$, thus $b \in F$. Suppose then 
that only $x_{1} \in I(F)$. Consequently, $(8,10)$ or $(7,11)$ is in $F$. However, the first choice is impossible and thus $(7,11) \in F$.

Let now $c^{\prime}=(9,9)$ and $f=(7,9)$. If $f \notin I(F)$, then $(9,10) \in F$. On the other hand, if $f \in I(F)$, we conclude that $b \in F$ since $c,(9,10)$ and $b$ must be covered by exactly one point of $F$. The cases $c^{\prime}=(9,8)$ and $c^{\prime}=(9,7)$ are trivial because around $c$ there is at least one element of $F$, and $c^{\prime}$ and the codeword of $I(F)$ just above it must be covered by one point of $F$ and thus the point above $c^{\prime}$ belongs to $F$.

(vi) Assume finally that $c=(9,6)$. Denote $d=(10,6)$. Let first $c^{\prime}=c$. Notice that $a$ or $d$ is in $F$. Denote further $f=(7,6)$. If $f \notin I(F)$ it is immediate that $d \in F$. Suppose $f \in I(F)$. Let $x_{1}=(7,7), x_{2}=(5,6)$ and $x_{3}=(7,5)$. If none of them is contained in $I(F)$, then $a \in F$. Now we have to check the cases where (at least) one of the $x_{i}$ 's is in $I(F)$. Suppose $x_{2} \in I(F)$. Now a point of $F$ must cover $x_{2}$. We get $a \in F$ except if $I(F)=\left\{x_{2}, f, c\right\}$ in which case $(6,6) \in F$. Let $x_{1} \in I(F)$. The case $I(F)=\left\{x_{1}, f,(7,8), c\right\}$ gives $x_{1} \in F$ and $I(F)=\left\{x_{1}, f, x_{3}, c\right\}$ implies that $f \in F$. For other sets $I(F)$ containing $x_{1}$ we know that $a \in F$. Assume now that $x_{1} \notin I(F)$. Let $x_{3} \in I(F)$. We obtain $x_{3} \in F$ if $I(F)=\left\{(7,4), x_{3}, f, c\right\}$ and $a \in F$ otherwise.

Suppose then that $c^{\prime}=b$. Let $f=(7,5)$. The situation $f \notin I(F)$ implies that $c^{\prime} \in F$. Assume now $f \in I(F)$. Does any of the points $x_{1}=(7,6), x_{2}=(5,5)$ or $x_{3}=(7,4)$ belong to $I(F)$ ? If not, then $(8,5) \in F$. If $x_{2} \in I(F)$ or $x_{3} \in I(F)$, then $c^{\prime} \in F$. Assume that only $x_{1}$ is in $I(F)$ of these three points. Now since $f \in I(F)$, either $(8,5) \in F$ or $x_{1} \in F$. If $(7,7) \notin I(F)$, then $(8,5) \in F$. The case $(7,7) \in I(F)$ implies that $x_{1} \in F$ because neither $f$ nor $(7,7)$ can be covered by the point in $F$ that covers $c$.

Let next $c^{\prime}=(9,3)$ and $f=(7,3)$. If $f \notin I(F)$, then $(9,4) \in F$. Assume hence that $f \in I(F)$. Then one element of $F$ covers $f$ and in order to cover both $c$ and $b$ we must have $b \in F$. It is very easy to see that for the same reason the cases $c^{\prime}=(9,2)$ and $c^{\prime}=(9,1)$ give $b \in F$. This completes the proof.

Theorem 6. There exists a weakly $(1, \leq 3)$-identifying code with density $\frac{7}{12}$ in the infinite square lattice.

Proof. We claim that the code of Fig. 6 with density $\frac{7}{12}$ is $(1, \leq 3)$-identifying.

We are given a pattern $I(F)$ and should always demonstrate that we can tell at least one element of $F$-clearly, $I(F)$ is empty if and only if $F$ is empty. Recall that the leader of $I(F)$ is the (unique) point in $I(F)$ with the highest value of the ordinate among the points with the highest value of the abscissa. By symmetry, it suffices to assume that the leader is among $c 2-c 9, d 1-d 5$ and $d 7$ (see Fig. 6 for the chessboard coordinates, which we use in parallel with the usual $(x, y)$-coordinates). Throughout the proof we call all the points in $B_{1}(v)$ the neighbours of $v$, i.e., we (a bit misleadingly) include $v$ itself among the neighbours.

Step 1: The leader is $c 2, c 3, c 4, c 5, c 6$ or $c 8$. These cases are immediate (see Example 1). If the leader is $(i, j)$, then in each of these cases $(i, j+1) \notin I(F)$ and $(i+1, j-1) \notin I(F)$ and the only neighbour of $(i, j)$ than can be in $F$ is $(i-1, j)$.

Step 2: The leader is $c 7 \in I(F)$. Then $b 7$ or $c 6 \in F$. If $c 6 \notin I(F)$ or $c 5 \notin I(F)$, then we can tell that $c 6 \notin F$ and consequently $b 7 \in F$, and we are done. We can therefore only consider cases in which $c 6, c 5 \in I(F)$. 


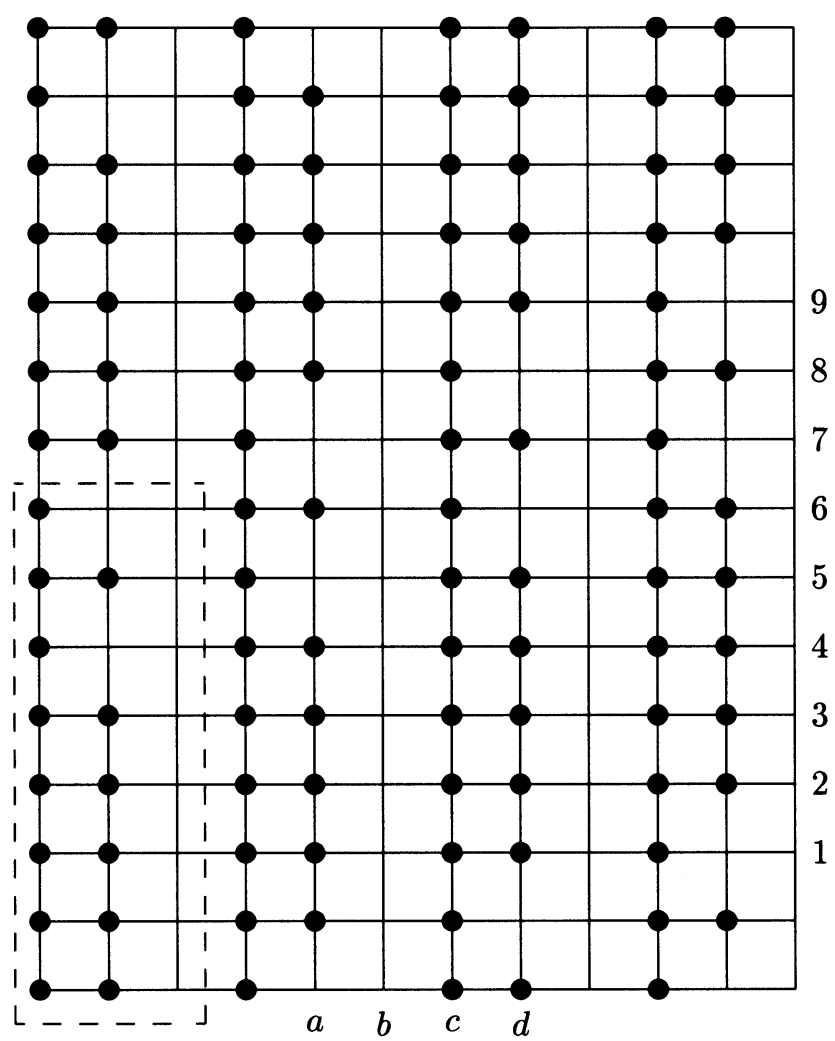

Fig. 6. A weakly $(1, \leq 3)$-identifying code with density $\frac{7}{12}$.

Consider the patterns obtained when $c 6 \notin F$. The fact that $c 6 \in I(F)$ now implies that $b 6 \in F$; and $c 5 \in I(F)$ implies that $b 5 \in F$. Hence $I(F)=\{c 7, c 6, c 5, a 6\}$, but given this pattern, we can immediately tell that the only neighbour of $a 6$ that can be in $F$ is $b 6$.

Given any pattern $I(F) \supseteq\{c 7, c 6, c 5\}$ other than $\{c 7, c 6, c 5, a 6\}$ with leader $c 7$, we know that $c 6 \in F$.

Step 3: The leader is $c 9$. This is very similar to the previous case. We deduce that $c 8 \in F$ or $b 9 \in F$, and are immediately done, unless $a 9, c 8, c 7 \in I(F)$. Assuming that the only patterns obtained when $c 8 \notin F$ result from $F=\{b 9, b 8, b 7\}$ and $F=\{b 9, b 8, c 6\}$, and in both cases, given the corresponding $I(F)$, we can immediately tell that the only neighbour of $a 9$ that can be in $F$ is $b 9$. Given any other $I(F)$ we know that $c 8 \in F$.

Step 4: The leader is $d 1, d 2, d 3$ or $d 4$. Let the leader be the point $(i, j)$. We know that $(i-1, j) \in F$ or $(i, j-1) \in F$. We can only consider patterns in which $(i-1, j+1),(i-$ $1, j),(i-1, j-1) \in I(F)$ : otherwise we can immediately tell that $(i, j-1) \in F$. Consider the patterns obtained when $(i-1, j) \notin F$ (in which case $(i, j-1) \in F$ ). It again suffices to prove that each of the resulting patterns $I(F)$ will immediately give away at least one element in $F$. 
Because $(i-1, j)$ has a neighbour in $F$, we know that $(i-2, j) \in F$ or $(i-1, j-1) \in$ $F$; because $(i-1, j+1) \in I(F)$, we know that $(i-2, j+1) \in F$ or $(i-1, j+2) \in F-$ and if the leader is $d 1, d 2$ or $d 3$, the latter is not possible and we are already done (because in the resulting patterns $(i-3, j+1) \in I(F)$ has a neighbour in $F$, and it can only be $(i-2, j+1))$. So assume that $(i, j)=d 4$. If $b 4=(i-2, j) \in F$, the two possibly resulting patterns $I(F)$ tell us that $b 4$ must be in $F$; if $c 3=(i-1, j-1) \in F$, then $c 2 \in I(F)$ and of its neighbours only $c 3$ can be in $F$.

Step 5. A similar argument to that presented in Step 4 shows that if $d 2, d 3, d 4$ or $d 5$ is the point in $I(F)$ with the lowest value of the ordinate among the points with the highest value of the abscissa, then all the resulting patterns $I(F)$ give away at least one element of $F$.

Step 6: $d 7$ is the leader. Then $d 7, d 6$ or $c 7 \in F$. Furthermore, it suffices to consider the patterns in which also $c 7$ and $c 6$ are in $I(F)$ : otherwise only one of $d 7, d 6$ or $c 7$ can be in $F$.

If $d 5 \notin I(F)$, then we know that $d 7 \in F$ or $c 7 \in F$, and we are done unless also $c 8$ is in $I(F)$. Again considering only the patterns resulting when $c 7 \notin F$ (in which case $d 7 \in F$ ) we see that $b 8 \in F$ or $c 8 \in F$, and the third element of $F$ is $b 6$ or $c 6$; but in all such patterns we immediately recognize that $b 8 \in F$ or that $c 8 \in F$.

It therefore suffices to consider the patterns with $d 5 \in I(F)$. Then among the neighbours of $d 5, d 7, c 7, c 6 \in I(F)$ at least two belong to $F$. By Step 5, we can assume that the lowest point $v$ of $I(F)$ in the column $d$ is $d 1$ or lower: but $v$ immediately identifies itself, as we know that there are two other members of $F$ but they are sufficiently far above $v$.

Step 7: $d 5$ is the leader. As in Step 6, we see that $d 5, d 4$ or $c 5 \in F$, and that it suffices to consider the patterns in which $d 5, d 4, c 5$ and $c 4$ are all in $I(F)$. Clearly,

$$
\left|\left(B_{1}(d 5) \cup B_{1}(d 4) \cup B_{1}(c 5) \cup B_{1}(c 4)\right) \cap F\right| \geq 2 .
$$

By Step 5, we can assume that the lowest point $v$ of $I(F)$ in the $d$-column is $d 1$ : if it is $d 2, d 3$ or $d 4$, we are immediately done by Step 5; if it is below $d 1$, then as in Step 6, $v$ immediately identifies itself, as we know that there are two other elements in $F$, but they are sufficiently far above it. We can only consider patterns in which $d 1, d 2, c 1$ and $c 2$ all belong to $I(F)$; otherwise, there is only one neighbour of $d 1$ which can belong to $F$. Clearly,

$$
\left|\left(B_{1}(d 2) \cup B_{1}(d 1) \cup B_{1}(c 2) \cup B_{1}(c 1)\right) \cap F\right| \geq 2 .
$$

By (2) and (3), $c 3 \in F$ or $d 3 \in F$, and hence all the ten points $c 1-c 5, d 1-d 5$ are in $I(F)$. If $c 3 \in F$, this is only possible if $d 5$ and $d 1$ are also in $F$, and then $I(F)$ consists of these ten points. If $d 3 \in F$, the only possibility is that also $c 1$ and $c 5$ are in $F$, in which case $I(F)$ consists of 12 points, so we are done.

\section{References}

1. I. Charon, I. Honkala, O. Hudry and A. Lobstein, General bounds for identifying codes in some infinite regular graphs. Electron. J. Combin. 8 (2001), R39. 
2. I. Charon, O. Hudry and A. Lobstein, Identifying codes with small radius in some infinite regular graphs. Electron. J. Combin. 9 (2002), R11.

3. G. Cohen, S. Gravier, I. Honkala, A. Lobstein, M. Mollard, C. Payan and G. Zémor, Improved identifying codes for the grid. Electron. J. Combin., Comments to 6(1) (1999), R19.

4. G. Cohen, I. Honkala, A. Lobstein and G. Zémor, New bounds for codes identifying vertices in graphs. Electron. J. Combin. 6(1) (1999), R19.

5. G. Cohen, I. Honkala, A. Lobstein and G. Zémor, On identifying codes, in Codes and Association Schemes (Proc. of the DIMACS Workshop on Codes and Association Schemes 1999), pp. 97-109. DIMACS Series in Discrete Mathematics and Theoretical Computer Science, Vol. 56. American Mathematical Society, Providence, RI, 2001.

6. T. W. Haynes, S. T. Hedetniemi and P. J. Slater, Fundamentals of Domination in Graphs. Marcel Dekker, New York, 1998.

7. I. Honkala, T. Laihonen and S. Ranto, On codes identifying sets of vertices in Hamming spaces. Des. Codes Cryptogr. 24 (2001), 193-204.

8. I. Honkala and A. Lobstein, On the density of identifying codes in the square lattice. J. Combin. Theory Ser. B 85 (2002), 297-306.

9. M. G. Karpovsky, K. Chakrabarty and L. B. Levitin, On a new class of codes for identifying vertices in graphs. IEEE Trans. Inform. Theory 44 (1998), 599-611.

10. T. Laihonen, Sequences of optimal identifying codes. IEEE Trans. Inform. Theory 48 (2002), 774-776.

11. D. F. Rall and P. J. Slater, On location-domination numbers for certain classes of graphs. Congr. Numer. 45 (1984), 97-106.

12. S. Ranto, I. Honkala and T. Laihonen, Two families of optimal identifying codes in binary Hamming spaces. IEEE Trans. Inform. Theory 48 (2002), 1200-1202.

13. P. J. Slater, Leaves of trees. Congr. Numer. 14 (1975), 549-559.

14. P. J. Slater, Locating dominating sets and locating-dominating sets, in Graph Theory, Combinatorics, and Applications (Proc. of the Seventh Quadrennial International Conference on the Theory and Applications of Graphs, eds. Y. Alavi and A. Schwenk), Vol. 2, pp. 1073-1079. Wiley, New York, 1995.

Received June 12, 2001, and in revised form June 18, 2002. Online publication October 29, 2002. 